\title{
DE
}

BULGARIAN ACADEMY OF SCIENCES

CYBERNETICS AND INFORMATION TECHNOLOGIES • Volume 15, No 2

Sofia • 2015

\section{A Survey of Solving Approaches for Multiple Objective Flexible Job Shop Scheduling Problems}

\author{
Krasimira Genova, Leoneed Kirilov, Vassil Guliashki \\ Institute of Information and Communication Technologies, 1113 Sofia \\ Emails:kgenova@iinf.bas.bg lkirilov@iinf.bas.bg vggul@yahoo.com
}

\begin{abstract}
Many real life scheduling problems can be formulated as Flexible Job Shop Scheduling Problems (FJSSPS) which simultaneously optimize several conflicting criteria. A typical feature of such problems is their high computational complexity. The purpose of this paper is to provide a review of the techniques, developed to solve multiple objective FJSSPs during the last decade. These techniques could be classified into two groups: approaches with application of mathematical models and heuristic approaches. Usually hybrid metaheuristic algorithms are proposed for large dimensional real life problems and they outlay the tendency for the future developments of efficient solution approaches for multiple objective FJSSPS.
\end{abstract}

Keywords: Scheduling problems, flexible job shop, multiple objectives, mathematical models, metaheuristic algorithms.

\section{Introduction}

The basic purposes of job shop schedules preparing are the execution of assigned volume jobs (activities) in deadlines, determined by the different client's orders (tasks) and the realization of these jobs with optimal economic indices for the production unit. The task of making a job shop schedule arises most often, when the available resources are restricted as a result of decisions, taken previously after a long time period planning and a short time period planning. Due to their wide spreading in different areas of human activity on one side, and to their 
combinatorial complex nature on the other side, the interest towards job shop schedules and sequencing problems in the international research centres has increased essentially in the recent years.

According to the jobs and operations to be processed, the number and types of machines that comprise the shop, and the disciplines that restrict the manner in which assignments can be made, the scheduling is classified as: a Single machine shop (one machine and $n$ jobs to be processed); Parallel machines shop (a number of one-operation jobs can be processed on any of the machines), Flow shop (in each job exactly one operation for every one machine, all jobs go through all the machines in the same order); Job shop (the operations of a job being totally ordered, each job can visit each machine twice or more often). The most common in practice are the Job Shop Scheduling Problems (JSSPs). The Flexible Job Shop Scheduling Problems (FJSSPs) are an extension of the classical JSSPs. They reflect the possibilities for flexibility of one production system, where one operation can be executed on different machines or one machine can execute different operations by means of a quick setup (for example tool-changing or jig-changing devices) [46]. In the first case this is achieved by having a set of identical machines, and in the second case - by high-tech multipurpose machines.

In creating applicable models of job shop schedules, a question arises if the constraints for some parameters should be introduced for achievement of determined purposes or these parameters should be included like criteria, which have to be optimized [45]. The constraint introduction fixes values, which cannot be violated and this may lead to impossibility a feasible solution to be found. On another side, using the criteria gives some tolerance for the task parameters and can guarantee the obtaining of a feasible solution. Taking into account several criteria during the evaluation of job shop schedules gives the possibility for one more realistic solution to be presented to the users.

The JSSPs are combinatorial and NP-hard problems. The FJSSPs are a much more complex version of the JSSPs, because of the additional need the assignment of operations to machines to be determined. Furthermore, they are strongly NP-hard. The computational difficulties increase in solving such problems in multiple objective context. These problems represent a challenge for the researchers in different international scientific research organizations. In recent years the need of developing new approaches for solving multiple objective FJSSPs has been intensified.

The published approaches during recent years for solving different classes of multi-objective problems for job shop schedules have been analyzed in several survey papers $[15,20,31,44]$. In the latest work [20] more than hundred publications have been systematized, connected with three groups of deterministic scheduling problems: single machine and parallel machines, flow shop, job shop and uncertain scheduling problems. The results from application of different approaches to each class of scheduling problems considered have been analyzed. The new trends in multiple objective scheduling have been discussed. In [46] a short review is made of different groups of approaches for solving multi-objective FJSSPs, published during the last ten years. 
The purpose of this paper is to make a state-of-the-art survey of the publications, devoted to optimization of multi-objective FJSSPs in the recent years. Their number is still small in comparison to the number of works, connected with other classes of job scheduling problems. But the trend of increasing interest towards these problems in the last time can be easily traced out. The paper is organized as follows: In Section 2 the formulation of multi-objective flexible job shop scheduling problems is discussed. In Section 3 two groups of solution methodologies for these optimization problems are presented and a review of the methods and algorithms published in the last decade is done. In the last section the characteristics of the published studies and the directions for future work are generalized.

\section{Formulation of multi-objective flexible job shop problems}

The classical JSSP could be formulated as follows. There is a set of $n$ jobs and a set of $m$ machines. The set of all machines is noted by $M, M=\left\{M_{1}, M_{2}, \ldots, M_{m}\right\}$. Each job $i$ consists of a sequence of $n_{i}$ operations. Each operation $O_{i, j}(i=1,2, \ldots, n$; $j=1,2, \ldots, n_{i}$ ) of job $i$ has to be processed on one machine $M_{k}$ out of a set of given machines $M$. There is a predefined set of processing times for each operation $O_{i, j}$ on a given machine $-t_{i, j, k}$. The FJSSPs are an extension of the classical JSSPs taking into account the production flexibility. Unlike the classical JSSP where each operation is processed on a predefined machine, each operation in the FJSSP can be processed on one $M_{k}$ out of several machines $M_{k} \in M_{i j}, M_{i j} \subseteq M$. If $M_{i j} \subset M$ for at least one operation, then it is a partial flexibility of JSSP (P-FJSSP); while if $M_{i j}=M$ for each operation, then it is a total flexibility of JSSP (T-FJSSP).

The minimization of the maximal completion time of all jobs (makespan) is the most common used performance criterion for FJSSPs. The minimization of a makespan is often combined with the minimization of the total workload of machines (sum of the working times over all machines) and the minimization of the maximal machine workload (sum of the processing times of operations on a critical machine) in multi-objective FJSSPs. Other used objectives are, for example: the maximal tardiness, maximal lateness, weight or mean completion time, weight number of tardy jobs, maximal cost.

In the process of solving FJSSPs the following assumptions are most often made: none of the operations cannot be interrupted during its processing (nonpreemption condition); there are no precedence constraints among operations of different jobs; each machine can perform only one operation at any time (resource constrain); each machine is available to other operations immediately after the last assigned operation to be completed; all machines are available at the start of the production process; the machines are independent from each other.

The problem of scheduling jobs in FJSSPs could be decomposed into two subproblems: the routing (assignment) sub-problem that assigns each operation to a 
relevant machine selected from a set of capable machines $M_{k} \in M_{i j}$, and the scheduling (sequencing) sub-problem that has to calculate the starting times of the assigned operations on all machines in order to obtain a feasible schedule (the technological constraints not violated) to minimize the predefined objective functions.

\section{Solution approaches for multi-objective flexible job shop problems}

The job shop scheduling problems are combinatorial and difficult computational problems. Optimal solutions can be found in polynomial time only for scheduling problems with a small number of jobs and machines [12, 19]. The FJSSPs are a much more complex version of the classical JSSPs, because of the additional need for the assignment of operations to machines to be determined. Furthermore they are strongly NP-hard. The combining several conflicting criteria for evaluating the efficiency of the generated job shop schedules leads to additional computational complexity.

In literature there are two approaches proposed for multi-criteria FJSSPs [46]. In the first approach the mathematical models of FJSSPs are formulated in terms of Mixed-Integer Programming (MIP). Conventional techniques for searching optimal solutions can be applied to these models, but they have still limited computational potentialities regarding the size of the problems, in spite of the increased computational capacity of the modern specialized MIP solvers and the essential computational resources of the modern computer systems. For problems of a larger size, different approximate and heuristic algorithms designed to solve these mathematical models are proposed.

The heuristic approaches, applied directly to the FJSSPs have much greater spreading in the references, for example: genetic algorithms, evolutionary algorithms, as well as other metaheuristics. The manners for coding the distinct solutions (schedules) play an essential role for the efficiency of these techniques in finding good feasible schedules.

For the development of heuristic algorithms, two types of approaches are applied: hierarchical and integrated. When using hierarchical approaches, the routing and scheduling sub-problems are consecutively solved, while in integrated approaches, the routing and scheduling sub-problems are not differentiated. The application of a decomposition approach reduces the complexity of solving the subproblem in flexible job shop scheduling.

\subsection{Solution approaches with application of mathematical programming models}

The mathematical models of the multiple objective FJSSPs, which are published in literature, are constructed in terms of multi-criteria mixed-integer linear or nonlinear mathematical programming. Very often they are extensions of previously published single objective models. Mathematical models are formulated also for problems, where additional specific constraints and assumptions are added to the classical formulation of multi-objective FJSSPs. 
In [28] the multi-objective FJSSP is considered, and the aim is to find a compromise schedule, that minimizes the mean flow time, the mean job tardiness, and the mean machine idle time of the manufacturing system to be found simultaneously. The authors suggest a mathematical model, which could be applied separately for each one of the three objective functions. For this model the planning horizon $T$ is defined in advance and the makespan of the schedule $\left(C_{\max }\right)$ cannot be greater than $T$. To find a trade-off schedule, a multiple-decision-making technique the global criterion method is proposed. For FJSSP test examples with a small size this method can find exact Pareto optimal solutions. For medium and large size test examples, the mathematical model size increases drastically and the application of the global criterion method is not acceptable.

In [9] a mathematical model of FJSSP with non-fixed availability constraints and minimizing of three criteria: makespan of the jobs; maximal machine workload and total workload of the machines is formulated as a model of mixed integer nonlinear programming. This model is generated with the aim for clear presenting the parameters and the constraints of the considered scheduling problem and a hybrid algorithm combining a Genetic Algorithm (GA) and local search is suggested for finding efficient schedules.

In [7] a multi-objective FJSSP with objectives to minimize the makespan and the total weighted tardiness is considered. The authors formulate a mixed-integer linear programming model for this multi-objective FJSSP which allows operations overlapping. This model is a further development of the singe objective model, proposed by [6]. This model consists of 12 groups of constraints and of a considerable number of integer and binary variables. In [8] a mathematical model of the problem of multi-objective dynamic scheduling in FJSSP is formulated. The efficiency and stability factors are considered in evaluating the solutions. These factors are measured by the objectives of minimization of the makespan, the starting time deviation and a penalty function of the total deviation. The model is a development of the model proposed in [6] in order to present an integrated representation of the dynamic scheduling in flexible job shop systems. But it is quite difficult to solve such models for medium and actual size problems with the help of traditional optimization approaches.

In [25] the production process of the seamless steel tube is considered, in which because of the availability of several identical machines in parallel, there exists flexibility of the production scheduling. For this reason the job shop scheduling problem is considered as FJSSP. This production is characterized as multiple stages with several parallel machines at each stage. There are two tasks of scheduling: one is to determine which jobs are allocated to every machine (determine the flexible production route of jobs) and the other is to determine the sequence of jobs at each stage. For this problem a non-linear mixed integer programming model is developed, where the total idle time of machines and the waiting time of jobs should be minimized, and a modified genetic algorithm is proposed.

In $[21,54]$ mathematical programming models for multiple objectives FJSSP (total or partial) are formulated. Three criteria should be minimized: the maximal 
completion time of machines (makespan); the maximal machine workload; and the total workload of machines. These models can be solved by means of traditional techniques for multi-objective optimization - weighted sum, $\varepsilon$-constraints or goal programming. Most often a generalized objective function is formulated as a weighted combination of the three criteria, where the weights can be generated in different manners. Nevertheless, for problems of a larger size, hybrid heuristic algorithms are suggested to find out solutions, closely located to the Pareto optimal solutions.

The mathematical model for the multi-objective FJSSP with Preventive Maintenance (PM) activities is defined in [24]. This model is similar to the model, suggested in [33] to which non-overlapping constraints were added between PM tasks and operations and the constraints ensure that the PM tasks have to be completed within their time windows. Again a heuristic algorithm is proposed to solve this model. It is a Discrete Artificial Bee Colony (DABC) algorithm.

In [18] the multi-objective FJSSP with overlapping in operations is studied. Because the makespan is not a good evaluation criterion with overlapping in operations assumption, the authors used in addition the total machine work loading time and the critical machine work loading time as evaluation criteria. For this model a Mixed Integer Linear Programming (MILP) model is formulated. Using Lingo software, the MILP model is solved with the help of the branch and bound method for test examples with a small size.

In [34] upon transforming the problem into an equivalent network problem, two mixed integer goal programming models are formulated to solve one generalization of the FJSSP. The generalization concerns the set-up times of the machines when they pass from one operation to another. The optimization criteria are executed in the following priority order: minimizing the makespan is the primary, balancing the workloads of the machines is the secondary goal. Both models have a relatively large number of variables and are suitable for small size problems. If the sequence dependent setup times are eliminated or replaced by the average times that are spent for visiting the job nodes, the size of the models can be reduced considerably.

A model of FJSSP with a limited number of fixtures and with an objective function minimizing the weighted sum of the completion times and tardiness for all jobs is proposed in [39]. This is a time-indexed model, where the suitable choice of the value for parameter $T$ is very important. This parameter determines the number of intervals, into which the planning horizon of the schedule is divided. For great $T$ the number of variables and constraints of the model increases considerably. The smaller values of $T$ lead to smaller computation times, but the quality of the schedule obtained gets worse. Thornblad et al. determine a suitable value of $T$ using heuristics. There are presented results from solving this model for problems with a realistic size, using standard optimization software, in acceptable computation times. In [40] and in the papers attached to it [41-43] other variants of the timeindexed model for FJSSP are presented taking into account additional constraints, including side constraints regarding the maintenance, fixtures and night shifts. For the first time objective weights are proposed as functions of the jobs' due dates, 
such that a delayed job is assigned a higher weight than a job that is less delayed. The presented computational results show that the proposed iterative procedure is an efficient approach to produce optimal, or near-optimal schedules for real data instances within a time frame which is acceptable in practice.

Demir and Isleyen [5] have made a review of the published mathematical models, designed for FJSSPs. They evaluate the mathematical models in respect of the decision variables used in them to represent the sequence of operations execution on the machines. Depending on these variables Demir and Isleyen are grouping the mathematical models in three groups: Sequence-position variable based model, Precedence variable based model and Time-indexed model, and they compare them with respect of the obtained values of the objective function, minimized makespan $C_{\max }$, CPU time, number of variables and constraints. The authors used test examples of a small and medium size, as well as conventional solvers for MIP models. Based on the results obtained, they recommended using precedence variable based models of the type, proposed in [33]. Among the totally 22 presented publications, bi- or multi-criteria models for FJSSPs are presented only in [8]. It is noted, that the mathematical programming formulation and the application of traditional techniques to find optimal solutions of FJSSP is an inefficient approach because of its NP-hard complexity, but this is a key-step to study the structure of such problems and to develop efficient heuristics for their optimization.

Really, one of the basic reasons for the development of mathematical models for multi-objective FJSSP and their solution by means of exact methods is the possibility to obtain solutions for problems with small and medium size. These solutions can be used as a comparison basis for the development of heuristic methods. This is achievable by means of good integer programming models in combination with good solvers for their solving. The traditional approaches in the multiple objectives' optimization that are used to solve them are the weighted sum, $\varepsilon$-constraint or goal criterion methods.

\subsection{Solution approaches with heuristics and meta-heuristics}

The second larger group of algorithms for solving multi-objective FJSSP developed recently is based on using heuristic and meta-heuristic approaches. These algorithms find one or a set of approximated Pareto optimal solutions for a reasonable computation time. Most of these algorithms are population based. The choice of suitable forms of solutions encoding is a crucial factor for its convergence and speed of the algorithms. The chosen form of encoding can ensure the work of the algorithm with feasible solutions only and the selecting of perspective solutions. Some of the meta-heuristic approaches using to solve multi-objective FJSSP are: genetic and evolutionary algorithms, particle swarm optimization and ant colony optimization algorithms. The heuristics local search, simulated annealing and tabu search are often applied in combination with population based algorithms in order to achieve faster approximation of Pareto optimal solutions. The hybrid approach often outperforms the algorithms operating alone. 
Two types of approaches are applied when developing heuristic algorithms: hierarchical (decomposition) approach and integrated one respectively. When using hierarchical approaches, the routing and scheduling sub-problems are solved consecutively, while in integrated approaches, the routing and scheduling subproblems are not differentiated. The application of a hierarchical approach reduces the complexity of solving a sub-problem in flexible job shop scheduling.

In [47] a hierarchical solution approach is proposed for solving multi-objective FJSSP. The objective is to minimize simultaneously the makespan, the total workload of machines, and the workload of the critical machine. The proposed approach makes use of the Particle Swarm Optimization (PSO) to assign operations to machines and the Simulated Annealing (SA) algorithm to schedule operations on each machine. Encoding is proposed for generating of the efficient solutions, where each particle's position value represents the priority level for each operation. During the hybrid search process, PSO provides initial solutions for SA. Each particle's fitness value is computed by SA algorithm. The fitness function is defined as a weighted sum of three criteria. PSO uses the solutions evaluated by SA to continue the evolution. By means of computational experiments it is demonstrated, that although this hybrid algorithm does not guarantee the optimality, it provides solutions with good quality in a reasonable time limit.

In [9] a hybrid Genetic Algorithm (hGA) for solving the multi-objective FJSSP is proposed. Three objectives are considered: makespan, maximal machine workload and total workload of the machines. Two vectors are used for chromosome encoding like in Gen and Cheng's method [13]. Namely, these are machine assignment vector for operations and operation sequence vector. This way of encoding ensures always a feasible sequence of operations and satisfaction of the technological restrictions. Note that here the times for machine prevention are not included. Thus the number of decision variables is reduced and consequently, the efficiency of GAs will be improved. The fitness function is formed based on a weighted sum of the three objectives with more preference to the makespan. Two kinds of efficient neighbourhood which only contains solutions that have possibilities to improve the initial solution are defined on the concept of a critical path. A local search procedure that works over the two kinds of neighbourhood is then hybridized with the genetic algorithm in order to enhance the search ability. The authors show on the basis of the experimental research the efficiency of their hGA with and without non-fixed availability constraints. In [10] a hGA is used again to solve multi-objective FJSSP's. In order to strengthen the search ability, the bottleneck shifting serves as a kind of a local search method under the framework of GA. The number of critical paths serves as an additional intermediate objective besides the three original criteria in order to guide the local search to the most promising areas. According to the authors, the balance between the genetic search and local search expressed as a balance between the depth search and broadness search within the bottleneck shifting is of great importance for improving the performance and efficiency of the hybrid genetic algorithm. Similar hybrid genetic approach is presented also in [14]. 
In [56] a heuristic algorithm, called multistage-based GA to solve total and partial FJSSP is presented. The considered objectives are the makespan, the total workloads of the machines and the maximum workloads of machines. The efficiency of every GA depends on the complexity of chromosome representation. Therefore, the authors suggest a multistage operation-based approach, denoting each operation as one stage, and each machine as one state. The multi-objective optimization GA (moGA) includes $K$ stages (the total number of operations for all the jobs), and $m$ states (the total number of machines). The efficiency of the suggested moGA is shown on the base of numerical experiments with practical data.

In [54] a hybrid Particle Swarm Optimization (hPSO) algorithm is presented. It combines evolutionary computation technique and tabu search strategy for local improvement. As a fitness function a weighted sum of three objectives is used: the makespan; the maximal machine workload; the total workload of machines. In this paper the $A$-string (machines) and $B$-string (operations) encoding is used to avoid the use of a repair mechanism. The elements of the strings, respectively, describe a concrete allocation of operations to each machine and sequence of operations on each machine. The authors apply their algorithm to solve problems ranging from a small scale to a large scale. The algorithm efficiency is shown in finding better schedules in a reasonable time.

In [55] an efficient GA is proposed. It is designed for solving FJSSP according to minimization of a makespan and balancing of the workload of the machines. The authors developed Global Selection (GS) and Local Selection (LS) methods for generating high-quality initial population. Also the quality and speed for finding near optimal solutions are improved. A new efficient encoding scheme of the individuals which takes into account all constraints of FJSSP is used. The chromosome representation has two components: Machine Selection and Operation Sequence (called MS and OS), and it requires no repair mechanism. A new selection and mutation operators are suggested in accordance with this encoding.

In [28] a hybrid heuristic algorithm for solving multi-objective FJSSPs with a medium and large size is proposed. A schedule generator, called ESCH (L o w and $\mathrm{Wu}$ [27]) for finding an initial solution is used. The procedures are applied in a consecutive way. The first, called a Sequence-Improving Procedure (SIP), is used to determine a better performance schedule from a certain routing plan; the other, called a Routing-Exchange Procedure (REP), is designed to select a favorable one from a large candidate pool. Meta-heuristic procedures like Simulated Annealing (SA) and Tabu Search (TS) are applied for local improvement. A Performance evaluation index is defined for evaluation of the best neighborhood. It considers the distance to the best values for each objective (mean flow time, mean job tardiness, and mean machine idle time) simultaneously. The experimental results for four combinations of meta-heuristics SA and TS and procedures SIP and REP are presented. It is shown that the combination SA/TS hybrid heuristic is more suitable than the other three in terms of both accuracy and efficiency in solving medium and large size problems. 
Tay and Ho [38] solve multi-objective FJSSP by simultaneously minimization of the makespan, the mean tardiness, and the mean flow time by means of Composite Dispatching Rules (CDRs). They suggest a genetic programming framework for generation of CDRs. The experimental results show that the generated CDRs outperform the single dispatching rules and the composite dispatching rules selected from literature over large sets of FJSSPs.

In [25] a Modified GA (MGA) for solving multi-objective FJSSP is proposed with application to the production of the seamless steel tube presentation. This MGA is developed on the basis of the formulated non-linear Mixed Integer Programming (MIP) model with consideration of maintenance planning, intermediate inventory, and parallel work machines. They apply appropriate encoding of chromosomes and genetic operators in order to obtain near optimal solution.

In [26] a Multi Particle Swarm Optimization (MPSO) approach is presented. The considered objectives are: (minimize) the sum of the completion times (flowtime) and the maximum completion time (makespan). The authors suggest encoding the representations of schedules by considering the particle's position. In their PSO algorithm, the position representation of particles has two components: the operation order and machine selection, and it is encoded using a variable length strategy. The different individual is separated into a different group to map the operation order and machine selection respectively, which is favorable and reasonable. To match the two component characteristics, the authors introduce a multi-swarm search algorithm. The results from the corresponding computational experiments indicate that the proposed algorithm is an efficient approach for the multi-objective FJSSP, especially for large scale problems.

$\mathrm{X}$ ing, Chen, Yang [48] propose an efficient search method for multiobjective FJSSP with three objectives: the makespan, the total workload of the machines and the critical machine workload. They construct a knowledge-based heuristic algorithm by integrating some empirical knowledge into a heuristic searching algorithm. The method is compared with eight other algorithms. The computational results demonstrate that this approach outperforms most of the eight cited algorithms in finding better optimal (near optimal) values for a number of 15 benchmark instances when using different weighting coefficients for the three objectives. In another paper, [49] a simulation model framework for obtaining an optimal or near-optimal production scheduling for a class of manufacturing problems is proposed. The framework consists of 6 subsystems: Input subsystem, Operation assignment subsystem, Operation sequencing subsystem, Objective evaluation subsystem, Control subsystem and Output subsystem. The operation assignment subsystem generates a feasible assignment of operations to each machine and optimizes it considering the total workload of machines and the critical machine workload. An Operation Assignment Machine Knowledge (OAMK) is used to improve the simulation model in this subsystem. By the OAMK accumulative knowledge, a given operation could be assigned to a more appropriate machine. In Operation sequencing subsystem the random method is applied to arrange each operation on every machine. In order to improve the sequencing 
performance, the authors apply Ant Colony Optimization algorithm (ACO) to provide an appropriate initial schedule for the next local search algorithm. The objective evaluation subsystem transforms the sequencing of operations on machines into a schedule. They evaluate the schedule thus achieved by the weighted sum of three objective functions. The main function of the control subsystem is the computational flow control and feasibility validation of the current solutions. The function of the output subsystem is to output some essential optimization results to users and the Gantt chart of the optimal schedule. This simulation model framework could be used by other researchers to improve the available algorithms or to add new ones.

In [32] a new method based on multi-objective PSO for solving FJSSP with three objectives, minimizing makespan, total machine workload and the biggest machine workload is proposed. The method of the weighted sum with randomly generated weighting coefficients is used for scalarization. A particle is presented in the form of a binary group. Elite reserved strategy and dynamic neighborhood operators are used. The efficiency of the method is proven by computational experiments.

In [8] the problem for multi-criteria FJSSP with objectives: minimization of the makespan, starting time deviation and a penalty function of the total deviation is solved by means of a genetic algorithm. Two-vector representation, as well as a hybrid strategy combining transfer of a special number of the best chromosomes and a $n$-Size tournament selection method to choose chromosomes for reproduction are used. The algorithm proposed achieves the optimal solutions for small size problems and near optimal solutions for medium size problems. For large size problems the convergence of the algorithm decreases.

In [18] a decomposition approach for solving real multi-objective FJSSP with overlapping in operations problem is used in order to reduce the complexity of the problem solving. At the first stage, the assignment algorithm is applied. Its output is the initial solution of the scheduling algorithm. The assignment and scheduling algorithm was based on a metaheuristic SA algorithm. The generated solutions are according to the total objective function providing three different objectives with different weights.

Motaghedi-Iarijani, Sabri-Iaghaie, Heydari [30] proposed a multi-objective genetic algorithm to solve FJSSP. They consider the same three contradictory criteria: the makespan, the total workload of the machines and the maximal workload of the machines. The compromise programming is used as a multiple objective approach and a global criterion on the basis of the three distinct criteria is built. This global objective is applied for evaluation of the chromosomes in the population. Two vector representations $A$-string and $B$-string are used for chromosome encoding as in [54]. $A$-string represents the machines assigned to operations and $B$-string defines the sequence of operations. The hill climbing approach is used to improve the derived solutions of the GA. The test experiments have shown that the proposed hybrid algorithm performs better than the other algorithms cited by the authors. 
A hybrid algorithm is proposed in [1]. It generates an approximation to Pareto optimal solutions in reasonable time with three objective functions: minimization for the makespan, maximum workload and total workload of machines. The authors used an efficient combination of random solutions with the results from Tabu Search (TS), Simulated Annealing (SA) and hybrid TSA algorithms to generate the initial population for genetic algorithm. The elitist property of GA and the optimal combination of three basic genetic operators contribute for obtaining better solutions in comparison to other heuristic algorithms.

In [16] hybridization of an improved GA with SA algorithm is applied to FJSSP having two objectives: minimization of the makespan and total workload of all machines. The improved GA is used to assign each operation on a machine and SA algorithm is used to calculate the individual value of fitness. A matrix encoding of chromosomes is applied. The results of experiments demonstrate that this hybrid algorithm can provide solutions with good quality on a different scale and its convergence is faster.

Paper [29] presents an approach based on a hybridization of the particle swarm and Local Search algorithm (MOPSO+LS) to solve the multi-objective FJSSP with different release times. In this algorithm an integrated approach is used. Each particle consists of two parts: the part defining the routing policy of the problem (machine assignment to operations) and the part indicating the sequence of the operations on each machine (operations priority). In the proposed MOPSO an elitism strategy is applied and a "domination-free" archive is supported. The local search algorithm is applied to each particle in order to reassign machines to operations from the critical path in the solution obtained from the scheduling procedure. For the experimental investigations test examples having three criteria are used: makespan, total workload and max workload. The conclusion is that PSO represents an optimization approach with a great potential for finding suitable solutions of FJSSPs with medium and large dimension in a reasonable computational time.

The authors of [50] have developed a Hybrid Multi-Objective Evolutionary Approach (H-MOEA) to solve the FJSSP with minimization of three objectives: makespan, total workload and maximal workload. A modified crowding distance measure is introduced to maintain the diversity of individuals and a local search based on critical path theory is incorporated to guarantee the convergence to Pareto optimal solutions. Two-vector representation of the chromosomes as in [11] and permutation representation of the operation sequence are used. When compared to other recently published approaches, using several well-known benchmark instances, the proposed algorithm obtains near Pareto optimal solutions with better quality and/or diversity.

In [2] a multi-objective memetic algorithm to solve the FJSSP with three criteria is proposed. The criteria are correspondingly: minimization of the makespan, maximum machine workload, and total machine workload. They combine a genetic algorithm with a local search technique and apply dominancebased and aggregation-based fitness assignment approaches respectively. A chromosome encoding is applied, where each gene is a 3-tuple $(j, i, k)$, in which $j, i$, 
and $k$ represent the indices of the job, operation, and machine, respectively. Using testing with classic problem instances, the efficiency of this algorithm regarding the number and the quality of the obtained solutions is proven.

The authors of [3] again consider the multi-objective FJSSP with the criteria: the makespan, the total workload of the machines and the maximum workload of the machines. The authors proposed a Multi-Objective Evolutionary Algorithm (MOEA), which uses efficient genetic operators for diversification of the population and Pareto dominance relation to compare and evaluate the solutions. The 3-tuple encoding scheme is applied. The basic feature of the presented algorithm is its simplicity. This algorithm requires only two parameters, which have to be tuned. The efficiency of MOEA is proven on the basis of fifteen benchmark test examples, and the proposed algorithm has found $70 \%$ or more of the non-dominated solutions for each of these examples.

The Memetic Algorithm (MA) is also proposed in [52, 53] for the multiobjective FJSSP with the objectives to minimize the makespan, total workload and critical workload. The authors adapt the Non-Dominated Sorting Genetic Algorithm II (NSGA-II) [4] for the multi-objective FJSSP by using a well-designed chromosome encoding/decoding scheme and genetic operators. In the local search algorithm, attached to the modified NSGA-II, a hierarchical strategy is applied. This strategy handles the three objectives. It mainly considers minimization of the makespan, while the other two objectives are regarded in the choice and evaluation of all the possible actions that could generate the acceptable neighbour. A twovector encoding scheme is applied, using a machine assignment vector and an operation sequence vector, corresponding to the two subproblems in the FJSSP. Besides, experimental results on benchmark problems are presented, which are compared to the results of other algorithms specially proposed for the multiobjective FJSSP.

In [37] an efficient hybrid algorithm is proposed to find out Pareto optimal solutions of FJSSP having three objectives, which should be minimized simultaneously: the makespan, the maximal machine workload, and the total workload. The variant of PSO for discrete problems is used for global search and SA for local search respectively. To evaluate the fitness of the particles, a Pareto ranking and crowding distance method are applied. The experimental research proves the advantages of the proposed algorithm in terms of the number and quality of the non-dominated solutions compared to other algorithms in the references.

In [36] the Novel Hybrid Meta-Heuristic Algorithm (NHGASA) is introduced to solve multiple objective FJSSP. Three objective functions in this paper are minimized: the makespan, the workload of the most loaded machine and the total workload of all machines. The NHGASA is a combination of the Multi-Objective Genetic Algorithm (MOGA) and simulated annealing. The first initial population is generated by GA, and then the exact number of individuals in each population is improved by SA. Pareto optimal solution approach is used in the multi-objective genetic algorithm and the solutions are evaluated by three objectives during all the steps. In this algorithm the $A$-string and $B$-string encoding [54] is applied to each solution. The experimental results prove that the NHGASA algorithm overcomes 
the other approaches, solving the same benchmarks in a shorter computational time and with higher quality.

In the study in [21] a Hybrid Tabu Search Algorithm (HTSA) for multiobjective FJSSP is proposed. The performance criteria considered are the makespan, the total workload of machines and the workload of the critical machine. In the proposed HTSA a TS algorithm is used to produce the neighboring solutions in the machine assignment module and a Variable Neighborhood Search (VNS) algorithm to perform the local search in the operation scheduling component. New neighborhood search rules in both modules are applied. In addition, an efficient leftshift function is designed to decode a solution to an active schedule. In [22] a Hybrid Pareto-based Artificial Bee Colony (HABC) algorithm is proposed for solving the multi-objective FJSSP. In the artificial bee colony algorithm each food source is represented by two vectors - the machine assignment vector and the operation scheduling vector. The artificial bee is divided into three groups: employed bees, onlookers, and scouts bees. An external Pareto archive set is introduced to record the non-dominated solutions found so far. NSGA II [4] is introduced to divide the archived solutions into several levels according to their dominated solutions number. The experimental results on the benchmark instances and comparisons with other recently published algorithms show the efficiency and effectiveness of the proposed algorithm.

A hybrid discrete Shuffled Frog-Leaping Algorithm (SFLA) is developed for solving the FJSSP with minimizing three objectives in [23]. Several approaches are presented to construct the initial population with a high level of quality. Then each frog in the population is assigned to a corresponding memeplex according to the number of individuals who dominate it and then the number of frogs who are dominated by it. In the evolution process two well-designed crossover operators are introduced to share information among the best frogs and the worst frog. To memorize the non-dominated solutions found so far and increase the population diversity, they presented an external Pareto archive set. Several neighborhood structures were designed in the algorithm to direct the local search to more promising search space. In the hybrid algorithm, the balance of the capability of exploration and exploitation was considered. There are presented experimental results from the comparison of the proposed HSFLA with other recently published algorithms for solving the FJSSPs.

The main features of the proposed in [24] novel Discrete Artificial Bee Colony (DABC) algorithm for multi-objective FJSSPs are as follows: an efficient initialization scheme is introduced to construct the initial population with a certain level of quality and diversity; several problem-related neighborhood structures are designed; a self-adaptive strategy is adopted to provide opportunities to learn the producing neighboring solutions in different promising regions, whereas an external Pareto archive set is designed to record the non-dominated solutions found so far; a TS-based local search heuristic is applied to enhance the exploitation performance; a novel decoding method is also presented to tackle maintenance activities in the schedules generated. The proposed DABC algorithm is tested on a set of wellknown benchmark instances found in literature. 
In [57] a simple and easily extendable heuristic algorithm, based on a constructive procedure is presented to solve the FJSSP with typically used objectives (makespan, total workload of the machines, maximal machine workload) and Preventive Maintenance (PM) constraints. The PM periods are planned periods of unavailability of the machines, and the scheduler has to schedule them along with the jobs to be processed. This assumption brings the solving problem closer to the real manufacturing situations. The main purpose of this algorithm is to produce reasonable and applicable schedules very quickly. The approach proposed uses an accurate, relatively comprehensive and flexible criterion for scheduling job operations and PM operations and constructing a feasible high-quality solution. In this criterion, several factors affecting the quality of solutions are used and to each of these factors, a variable weight is assigned. By setting different values to these variable weights, different solutions are generated and evaluated. This heuristic method can be used to improve the quality of the initial feasible solution of the metaheuristics applied to solve the FJSSP, since the choice of a good initial solution is an important aspect of the performance of algorithms in terms of the computing time and solution quality.

In [35] an integrated heuristic approach is proposed to solve the multiobjective FJSSPs with simultaneous optimization of three objectives, including minimization of the makespan, total workload and maximum workload of machines. A meta-heuristic algorithm NSGA II (D e b et al. [4]) and two heuristic local search algorithms, based on the critical path method, are combined. The authors developed a floating search procedure applying local heuristic algorithms. Thus the solution of the problem is done in two sections, including assigning and sequencing sub-problems. First of all, search is done upon the assignment space achieving an acceptable solution and then the search will continue on the sequencing space. Pareto optimal solutions are found by means of an adapted version of the multi-objective algorithm NSGA II. Through a comparison study it is shown, that the algorithm proposed has been capable of producing better solutions.

In [51], in order to solve the same multi-objective FJSSP, a Quantum Immune Algorithm (QIA), based on the quantum and immune principles, is proposed. The authors use the weighted sum of three objectives to scalarize the multi-objective problem and a two parts encoding scheme with $A$-string and $B$-string. The quantum concept is used in initial random population. In the process of solving, the antibody is proliferated and divided into a set of subpopulation groups. The antibodies in a subpopulation group are represented by multistage gene quantum bits. In the antibody's updating, the general quantum rotation gate strategy and the dynamic adjusting angle mechanism are applied to accelerate the convergence. The QIA is efficient in solving MFJSSP and can be applied in other hybrid algorithms in the future.

A new population based metaheuristic solving multi-objective FJSSP with simultaneous minimization of three objectives (the makespan, the workload of the critical machine and the total workload of all machines) and with limited resource constraints, is presented in [17]. The authors have proposed a hybrid algorithm combining the Discrete Firefly Algorithm (DFA) and the local search approach. 
The standard firefly algorithm is a population based technique for solving continuous NP-hard problems. Therefore, in DFA the machine assignment and operation sequence are processed by constructing a suitable conversion of the continuous functions as attractiveness, distance and movement, into new discrete functions. Benchmark problems are used to evaluate the performance of the algorithm proposed.

From the last research publications, presenting metaheuristic approaches for solving multi-objective FJSSPs, several tendencies are outlined:

- hierarchical (decomposition) approach is more often applied with the aim to decrease the computational complexity of the implemented algorithm;

- combination of different heuristics are applied with the purpose to find better initial solutions and avoid the local optima;

- application of new, non-traditional metaheuristics;

- an attempt to simplify the algorithmic schemes with the aim of easy adapting when the assumptions and the constraints of the problem are changed.

\section{Conclusion}

By means of the multi-objective FJSSP's models various real-life problems can be presented. For this reason the interest of researchers from different areas of applied mathematics in the development of efficient algorithms to find out good Pareto optimal solutions in a reasonable computation time is great. From the recent publications it can be seen, that there are two main research directions: i) development of efficient mathematical models and suggestion of algorithmic approaches for their solving, and ii) direct use of different heuristic and metaheuristic approaches to find out near Pareto optimal solution in a reasonable time. Obviously, for large scaled multi-objective FJSSPs the second approach is the only suitable one. Here two fields arise for future research and developments. The first one is connected with the search of a good balance between the hierarchical and the integrated approaches. The hierarchical approach reduces the complexity of the algorithm by making the search space smaller. Therefore, some good solutions could be omitted because this job shop problem has been solved for a specific operations assignment found at the assignment step. However, changing the assignment may provide a better solution. The integrated approach considers both subproblems together and searches in two spaces. This pattern of searching tends to increase the variability in the search space. Thus, it is essential to design an approach for possible decreasing complexity of the problem by performing a complete search in the search space.

Another research field is connected with evaluation of the advantages of the developed metaheuristic algorithms. The computational results and comparisons with before published algorithms on public benchmark test instances are presented in every publication of a new metaheuristic algorithm. Typically, the new algorithms are presented by a set of very well calibrated parameter values, while other metaheuristic approaches included in the study for comparison are employed using a standard parameter setting. One way of measuring the quality of a 
metaheuristic approach [40] is to compute the average objective values found over a number of $N$ independent runs together with the "best of $N$ runs". In this way a measure of "how repetitive the proposed algorithm is" could be given, and the comparison between the metaheuristic algorithms being proposed to become more objective is made.

The development of fast and efficient algorithms solving multi-objective job shop scheduling problems remains a challenge for the researchers from different areas of applied mathematics.

Acknowledgments: This study is partially supported by Project No BG161PO003-1.1.06-0083 of the EU Operative Program "Development of Bulgarian economy competitiveness", entitled: "Scientific research for the purposes of development of software tool for generating efficient schedules by an innovative method for multiple objective optimization in discrete manufacturing within the scope of small and medium enterprises".

\section{References}

1. A z a rdoost, E. B., N. I man i pour. A Hybrid Algorithm for Multi Objective Flexible Job Shop Scheduling Problem. - In: Proc. of International Conference on Industrial Engineering and Operations Management, Kuala Lumpur, Malaysia, 22-24 January 2011, pp. 795-801.

2. Chiang, T., H. Lin. Flexible Job Shop Scheduling Using a Multiobjective Memetic Algorithm. - Advanced Intelligent Computing Theories and Applications with Aspects of Artificial Intelligence. Lecture Notes in Computer Science, Vol. 6839, 2012, pp. 49-56.

3. Chiang, T., H. Lin. A Simple and Effective Evolutionary Algorithm for Multiobjective Flexible Job Shop Scheduling. - Int. J. Production Economics, Vol. 141, 2013, pp. 87-98.

4. Deb, K., A. Pratap, S. Agarwal, T. Meyarivan. A Fast and Elitist Multiobjective Genetic Algorithm: NSGA-II. - IEEE Transactions on Evolutionary Computation, Vol. 6, 2002, No 2, pp. 182-197.

5. Demir, Y., S. K. I sle yen. Evaluation of Mathematical Models for Flexible Job-Shop Scheduling Problems. - Applied Mathematical Modelling, Vol. 37, 2013, pp. 977-988.

6. F a t t a hi, P., M. S. M e hrabad, F. J o l a i. Mathematical Modeling and Heuristic Approaches to Flexible Job Shop Scheduling Problems. - Journal of Intelligent Manufacturing, Vol. 18, 2007, No 3, pp. 331-342.

7. F a t t a h i, P. A Hybrid Multi Objective Algorithm for Flexible Job Shop Scheduling. - Int. J. Computational and Mathematical Sciences, Vol. 3, 2009, No 5, pp. 215-220.

8. F a t t a hi, P., A. F a llahi. Dynamic Scheduling in Flexible Job Shop Systems by Considering Simultaneously Efficiency and Stability. - CIRP Journal of Manufacturing Science and Technology, Vol. 2, 2010, pp. 114-123.

9. G a o, J., M. G e n, L. S u n. Scheduling Jobs and Maintenances in Flexible Job Shop with a Hybrid Genetic Algorithm. - J. Intell. Manuf., Vol. 17, 2006, pp. 493-507.

10. G a o, J, M, G e n, L. S u n, X. Z h a o. A Hybrid of Genetic Algorithm and Bottleneck Shifting for Multi-Objective Flexible Job Shop Scheduling Problems. - Comput. Ind. Eng., Vol. 53, 2007, doi:10.1016/j.cie.2007.04.010, pp. 149-162.

11. G a o, J., L. S u n, M. G e n. A Hybrid Genetic and Variable Neighborhood Descent Algorithm for Flexible Job Shop Scheduling Problems. - Computers and Operations Research, Vol. 35, 2008, No 9, pp. 2892-2907.

12. G arey, M. R., D. S. Johns on, R. S ethi. The Complexity of Flowshop and Jobshop Scheduling Scheduling. - Math. Oper. Res., Vol. 1, 1976, No 2, pp. 117-129.

13. G e n, M., R. C h e n g. Genetic Algorithms \& Engineering Design. NewYork, Wiley, 1997.

14. Gen, M., G. Jie, L. Lin. Multistage-Based Genetic Algorithm for Flexible Job-Shop Scheduling Problem. - Intelligent and Evolutionary Systems, Studies in Computational Intelligence, Vol. 187, 2009, pp. 183-196. 
15. H o o g e ve en, H. Multicriteria Scheduling. - European Journal of Operational Research, Vol. 167, 2005, Issue 3, pp. 592-623.

16. J i a n g J., M. W e n, K. M a a, X. L o n g, J. L i. Hybrid Genetic Algorithm for Flexible Job-Shop Scheduling with Multi-Objective. - Journal of Information \& Computational Science, Vol. 8, 2011, No 11, pp. 2197-2205.

17. Karthikeyan, S., P. A s okan, S. Ni ckolas. A Hybrid Discrete Firefly Algorithm for Multi-Objective Flexible Job Shop Scheduling Problem with Limited Resource Constraints. - International Journal of Advanced Manufacturing Technology, Vol. 72, 2014, Issue 9-12, pp. 1567-1579.

18. Khalife, M. A., B. A bbasi, A. H. K. D. Ab a di. A Simulated Annealing Algorithm for Multi Objective Flexible Job Shop Scheduling with Overlapping In Operations. - J. Ind. Eng. Vol. 5, 2010, pp. 17-28.

19. Lawler, E, .J. K Lenstra, A. H. G Rinnooy Kan, D. B Shmoys. Sequencing and Scheduling: Algorithms and Complexity. Chapter 9. - In: S. C. Graves et al., Eds. Handbooks in Operations Research and Management Science: Logistics of Production and Inventory. North-Holland, Amsterdam, 1993, pp. 445-522.

20. L e i, D. Multi-Objective Production Scheduling: A Survey. - International Journal of Advanced Manufacturing Technology, Vol. 43, 2009, Issue 9-10, pp. 926-938.

21. L i, J.-Q., Q.-K. P a n, Y. Li an g. An Effective Hybrid Tabu Search Algorithm for MultiObjective Flexible Job-Shop Scheduling Problems. - Computers \& Industrial Engineering, Vol. 59, 2010, pp. 647-662.

22. L i, J.-Q., Q.-K. P a n, S. X i e, S. W a n g. A Hybrid Artificial Bee Colony Algorithm for Flexible Job Shop Scheduling Problems. - Int. J. of Computers, Communications \& Control, Vol. VI, 2011, No 2, pp. 286-296.

23. Li, J.-Q., Q.-K. Pan, S. Xi e. An Effective Shuffled Frog-Leaping Algorithm for MultiObjective Flexible Job Shop Scheduling Problems. - Applied Mathematics and Computation, Vol. 218, 2012, pp. 9353-9371.

24. L i, J.-Q., Q.-K. P a n, M. F. T a s g e t i r e n. A Discrete Artificial Bee Colony Algorithm for the Multi-Objective Flexible Job-Shop Scheduling Problem with Maintenance Activities. Applied Mathematical Modelling, Vol. 38, 2014, pp. 1111-1132.

25. L i n, L., H. J i a-z h e n. Multi-Objective Flexible Job-Shop Scheduling Problem in Steel Tubes Production. - Systems Engineering - Theory \& Practice, Vol. 29, 2009, Issue 8, pp. 117-126.

26. L i u, H., A. A b r a h a m, Z. W a n g. A Multi-Swarm Approach to Multi-Objective Flexible JobShop Scheduling Problems. - Fundamenta Informaticae, Vol. 95, 2009, pp. 1-25, DOI: $10.3233 /$ FI-2009-184.

27. L o w, C, T-H. W u. Mathematical Modelling and Heuristic Approaches to Operation Scheduling Problems in an FMS Environment. - International Journal of Production Research, Vol. 39, 2001, No 4, pp. 689-708.

28. L o w, C., Y. Y i p, T.-H. W u. Modelling and Heuristics of FMS Scheduling with Multiple Objectives. - Comput. Oper. Res, Vol. 33, 2006, pp. 674-694.

29. Moslehi, G., M. Mahnam. A Pareto Approach to Multi-Objective Flexible Job-Shop Scheduling Problem Using Particle Swarm Optimization and Local Search. - Int. J. Production Economics, Vol. 129, 2011, pp. 14-22.

30. M o t a g h e d i-I a r i j a n i, A., K. S a b r i-I a g h a i e, M. H e y d a r i. Solving Flexible Job Shop Scheduling with Multi Objective Approach. - Int. J. of Industrial Engineering and Production Research, Vol. 21, 2010, No 4, pp. 197-209.

31. N a g a r, A., J. H a d d o c k, S. S. H e r a g u. Multiple and Bicriteria Scheduling: A Literature Survey. - European Journal of Operational Research, Vol. 81, 1995, pp. 88-104.

32. N a i-p i n g, H., W. P e i-l i. An Algorithm for Solving Flexible Job Shop Scheduling Problems Based on Multi-Objective Particle Swarm Optimization. -In: International Symposium on Information Science and Engineering (ISISE'2010), IEEE, 2010, pp. 507-511, DOI:10.1109/ISISE.2010.128.

33. Öz güven, C., L. Özbakır, Y. Yavuz. Mathematical Models for Job-Shop Scheduling Problems with Routing and Process Plan Flexibility. - Applied Mathematical Modelling, Vol. 34, 2010, pp. 1539-1548. 
34. Ö z g üve n, C., Y. Y a vu z, L. Ö z ba kır. Mixed Integer Goal Programming Models for the Flexible Job-Shop Scheduling Problems with Separable and Non-Separable Sequence Dependent Setup Times. - Applied Mathematical Modelling, Vol. 36, 2012, pp. 846-858.

35. S a d a ghi an i, J., S. B or o u j e rdi, M. Mirhabibi, P. S a d a g h i a ni. A Pareto Archive Floating Search Procedure for Solving Multi-Objective Flexible Job Shop Scheduling Problem. - Decision Science Letters, Vol. 3, 2014, No 2, pp. 157-168.

36. S h a h s a vari-P o ur a, N., B. G h a s e m i s h a b a n k a r e h. A Novel Hybrid Meta-Heuristic Algorithm for Solving Multi Objective Flexible Job Shop Scheduling. - Journal of Manufacturing Systems, Vol. 32, 2013, pp. 771-780.

37. Sh a o, X., W. Li u, Q. Li u, C. Z hang. Hybrid Discrete Particle Swarm Optimization for Multi-Objective Flexible Job-Shop Scheduling Problem. - International Journal of Advanced Manufacturing Technology, Vol. 67, 2013, Issue 9-12, pp. 2885-2901.

38. Tа y, J. C, N. B. Ho. Evolving Dispatching Rules Using Genetic Programming for Solving Multi-Objective Flexible Job-Shop Problems. - Computers \& Industrial Engineering, Vol. 54, 2008, pp. 453-473.

39. Thornblad, K., A.-B. Stromberg, M. Patriks son, T. Almgren. A Time-Indexed Formulation of a Flexible Job Shop Problem with a Limited Number of Fixtures. - In: Proc. of 13th International Conference on Project Management and Scheduling, 1-4 April 2012, Leuven, Belgium, 2012, pp. 335-338.

40. Thornblad, K. Mathematical Optimization in Flexible Job Shop Scheduling, Modelling, Analysis, and Case Studies. PhD Thesis, Chalmers University of Technology and University of Gothenburg, Göteborg, Sweden, 2013.

41. Thornblad, K., A.-B. Stromberg, M. Patriksson, T. Almgren. Scheduling Optimization of a Real Flexible Job Shop Including Side Constraints Regarding Maintenance, Fixtures, and Night Shifts, Accepted August 2013. http://www.optimization-online.org/DB_HTML/2013/08/3995.html

42. Thornblad, K., A.-B. Stromberg, M. Patriks son, T. Almgren. A Competitive Iterative Procedure Using a Time-Indexed Model for Solving Flexible Job Shop Scheduling Problems, Accepted August 2013. http://www.optimization-online.org/DB_HTML/2013/08/3991.html

43. Thornblad, K., A.-B. Stromberg, M. Patriks on, T. Almgren. Scheduling Optimization of a Real Flexible Job Shop Including Fixture Availability and Preventive Maintenance. - European Journal of Industrial Engineering, Accepted 2014. http://www.gu.se/english/research/publication/?publicationId=167036

44. T'kindt, V., J.-C. B illa ut. Multicriteria Scheduling Problems: A Survey. - RAIROOperations Research, Vol. 35, 2001, No 2, pp. 143-163.

45. T'k in d t, V., J.-C. B ill a u t. Multicriteria Scheduling - Theory, Models and Algorithms. Springer-Verlag, Berlin, 2006.

46. Wojakowski, P., D. Warzolek. Research Study of State-of-the-Art Algorithms for Flexible Job-Shop Scheduling Problem. - Technical Transactions, Mechanics, Vol. 1-M, 2013, pp. 381-388.

https://suw.biblos.pk.edu.pl/resources/i3/i8/i6/i4/i1/r38641/WojakowskiP_ResearchStud y.pdf

47. X i a, W., Z. W u. An Effective Hybrid Optimization Approach for Multi-Objective Flexible JobShop Scheduling Problems. - Computers \& Industrial Engineering, Vol. 48, 2005, pp. 409425.

48. X in g, L.-N., Y.-W. Ch e n, K.-W. Y an g. An Efficient Search Method for Multi-Objective Flexible Job Shop Scheduling Problems. - Journal of Intelligent Manufacturing, Vol. 20, 2009, pp. 283-293.

49. X i n g, L.-N., Y.-W. C h e n, K.-W. Y a n g. Multi-Objective Flexible Job Shop Schedule: Design and Evaluation by Simulation Modelling. - Applied Soft Computing, Vol. 9, 2009, pp. 362376.

50. X i ong, J., X. Ta n, K.-W. Y ang, L.-N. Xing, Y.-W. Chen. A Hybrid Multiobjective Evolutionary Approach for Flexible Job-Shop Scheduling Problems. - Mathematical Problems in Engineering, Vol. 2012, 2012, pp. 1-27, Article ID 478981, DOI:10.1155/2012/478981. 
51. X u e, H., P. Z h a n g, S h. W e i, L. Y a n g. An Improved Immune Algorithm for Multiobjective Flexible Job-Shop Scheduling. - Journal of Networks, Vol. 9, 2014, No 10, pp. 2843-2850.

52. Y u a n, Y., H. X u. A Memetic Algorithm for the Multi-Objective Flexible Job Shop Scheduling Problem. - In: Proc. of 15th Annual Conference on Genetic and Evolutionary Computation (GECCO’13), ACM, New York, 2013, pp. 559-566, DOI:10.1145/2463372.2463431.

53. Y u a n, Y., H. X u. Multiobjective Flexible Job Shop Scheduling Using Memetic Algorithms. Automation Science and Engineering, IEEE Transactions on, Vol. PP, 2013, Issue 99, pp. 1-18, DOI: 10.1109/TASE.2013.2274517.

54. Z h a n g, G., X. S h a o, P. L i, L. Ga o. An Effective Hybrid Particle Swarm Optimization Algorithm for Multi-Objective Flexible Job-Shop Scheduling Problem. - Computers \& Industrial Engineering, Vol. 56, 2009, Issue 4, pp. 1309-1318.

55. Zhang, G., L. Ga o, Y. Shi. An Effective Genetic Algorithm for the Flexible Job-Shop Scheduling Problem. - Expert Systems with Applications, Vol. 38, 2011, pp. 3563-3573.

56. Z h a n g, H. P., M. G e n. Multistage-Based Genetic Algorithm for Flexible Job-Shop Scheduling Problem. - Journal of Complexity International, Vol. 11, 2005, pp. 223-232.

57. Ziaee, M. An Efficient Heuristic Algorithm for Flexible Job Shop Scheduling with Maintenance Constraints. - Applied Mathematics and Sciences: An International Journal (MathSJ), Vol. 1, 2014, No 1, pp. 19-31. 